\title{
Analysis of NFKB2-mediated regulation of mechanisms underlying the development of Hodgkin's lymphoma
}

\author{
YUNPING ZHANG ${ }^{1}$, LAIGEN TONG $^{1}$, SISI CHEN ${ }^{1}$, WENZHONG WU ${ }^{1}$ and LI WANG $^{2}$ \\ ${ }^{1}$ Department of Hematology, The Affiliated Yixing Hospital of Jiangsu University, Yixing, Jiangsu 214200; ${ }^{2}$ Department of \\ Hematology, The First Affiliated Hospital of Nanjing Medical University, Nanjing, Jiangsu 210029, P.R. China
}

Received November 5, 2017; Accepted March 26, 2018

DOI: $10.3892 / \mathrm{mmr} .2018 .8911$

\begin{abstract}
Nuclear factor- $\kappa \mathrm{B}(\mathrm{NF}-\kappa \mathrm{B})$ is widely involved in various lymphoid malignancies. However, its exact functional role and potential regulatory mechanisms in Hodgkin's lymphoma (HL) remains unclear. The present study aimed to investigate the regulatory mechanism of NF- $\mathrm{KB}$ in $\mathrm{HL}$ by analysis of a gene expression profile that was obtained from HL cells with or without NF- $\mathrm{KB}$ subunit 2 (NFKB2) knockdown. The GSE64234 dataset containing 6 HL cell line specimens transfected with small interfering (si)RNA against NFKB2 and 6 control specimens transfected with non-targeting siRNA sequences was downloaded from the Gene Expression Omnibus database. Based on these data, differentially expressed genes (DEGs) were screened for following data preprocessing. Functional enrichment analysis was subsequently conducted among the identified upregulated and downregulated DEGs. Additionally, a protein-protein interaction (PPI) network was constructed and module analyses were performed. Finally, microRNAs (miRNAs/miRs) targeting the identified DEGs were predicted for the construction of a miRNA-target regulatory network. A total of 253 DEGs were identified, consisting of 109 upregulated and 144 downregulated DEGs. Pathway enrichment analysis revealed that B-cell lymphoma 2-like 1 (BCL2L1) was significantly enriched in the NF- $\kappa \mathrm{B}$ signaling pathway, and colony-stimulating factor 2 (CSF2) and BCL2L1 were enriched in the Jak-signal transducer and activator of transcription (STAT) signaling pathway. BCL2L1 and CSF2 were determined to be hub genes in the PPI network. A total of 6 miRNAs, including let-7a-5p, miR-9-5p, miR-155-5p, miR-135a-5p, miR-17-5p and miR-375, were identified in the miRNA-target
\end{abstract}

Correspondence to: Dr Li Wang, Department of Hematology, The First Affiliated Hospital of Nanjing Medical University, 300 Guangzhou Road, Gulou, Nanjing, Jiangsu 210029, P.R. China E-mail: wengfan72toudi@163.com

Key words: nuclear factor- $\kappa \mathrm{B}$ subunit 2, Hodgkin's lymphoma, differentially expressed genes, functional enrichment analysis, microRNA regulatory network. The results of the present study indicated that NFKB2 may be involved in HL development through regulation of BCL2L1, CSF2, miR-135a-5p, miR-155-5p and miR-9-5p expression, as well as the modulation of Jak-STAT and $\mathrm{NF}-\kappa \mathrm{B}$ signaling pathways.

\section{Introduction}

Hodgkin's lymphoma (HL) is a common malignant tumor among adolescents (1) that is divided into classical and nodular lymphocyte predominant variants based on the 2008 World Health Organization classification (2). Using chemotherapy and radiotherapy, alone or in combination, the durable remission rate for $\mathrm{HL}$ is $\sim 60-80 \%(3,4)$. However, survivors have an increased risk of long-term sequelae, including cardiovascular disease and secondary malignancy $(5,6)$. Therefore, understanding the underlying molecular mechanisms of $\mathrm{HL}$ development is essential for the discovery of novel therapeutic targets for HL.

Previous studies have demonstrated that the development and maintenance of various solid tumors and lymphoid malignancies is driven by nuclear factor- $\kappa \mathrm{B}(\mathrm{NF}-\kappa \mathrm{B})(7,8)$. Constitutive $\mathrm{NF}-\kappa \mathrm{B}$ activation has been identified in classical HL (9). A recent study by de Oliveira et al (10) investigated the functional roles and specific transcriptomes of $\mathrm{NF}-\kappa \mathrm{B}$ dimers with distinct subunit compositions by knockdown of NF-kB subunit 2 (NFKB2) in a HL cell line; it was reported that NFKB2 knockdown upregulated genes associated with hematopoietic and lymphoid organ development. NFKB2 is a member of the transcription factor complex $\mathrm{NF}-\kappa \mathrm{B}$ family, which promotes tumor cell proliferation and inhibits apoptosis (11). Truncations in the C-terminal region of NKFB2 are closely associated with tumorigenesis in various hematopoietic tumors, including multiple myeloma, chronic lymphocytic leukemia and cutaneous T-cell lymphoma $(12,13)$. A clear understanding of the regulatory role of NFKB2 in HL may contribute to the development of HL therapies.

de Oliveira et al (10) only analyzed the differentially expressed genes (DEGs) associated with NFKB2 knockdown in a HL cell line, and the regulatory mechanism of NFKB2 in HL remains poorly understood. Therefore, in the present study, the regulatory mechanism of NFKB2 in the tumorigenesis of HL was investigated by downloading the gene expression 
data (GSE64234) deposited by de Oliveira et al (10). DEGs between the NFKB2 knockdown and control groups were screened for prior to functional enrichment analysis of the identified DEGs, followed by protein-protein interaction (PPI) network and module analyses. Furthermore, microRNA (miRNA/miR) targets were predicted for the identified DEGs and a miRNA-target regulatory network was constructed.

\section{Materials and methods}

Affymetrix microarray data. The GSE64234 gene expression profile dataset deposited by de Oliveira et al (10) was downloaded from the Gene Expression Omnibus (https://www.ncbi.nlm.nih.gov/geo/) database, which was based on the GPL6244 Affymetrix Human Gene 1.0 ST Array (Thermo Fisher Scientific, Inc., Waltham, MA, USA). Data on $12 \mathrm{HL}$ cell line specimens were available in GSE64234, including 6 treated with non-targeting small interfering (si)RNA (control group) and 6 with NFKB2-targeted siRNA (knockdown group).

Data preprocessing. Probe data in CEL files were transformed into expression profiles. If numerous probes corresponded to the same gene, the mean expression value of the probes was used as the gene expression value. The oligo package (version 1.34.0) (14) in $\mathrm{R}$ (version 3.3.2) (15) was used to perform raw data format conversion, missing value imputation and background correction normalization for expression profile data. Quartiles were used for data normalization.

$D E G$ screening. The gene expression matrix derived from the knockdown and control groups was analyzed to identify the DEGs. P-values for genes were calculated with the non-paired t-test using the limma package (version 3.10.3) (16) in $\mathrm{R}$ and subsequently adjusted using the Benjamin and Hochberg method. An adjusted P-value of $<0.05$ was set as the threshold value. To directly assess DEG expression in these samples and confirm the classification, a DEG heat map was drawn using the pheatmap package (version 1.0.8) (17) in R.

Functional enrichment analyses. Upregulated and downregulated DEGs were evaluated based on Gene Ontology (GO) (18) and Kyoto Encyclopedia of Genes Genomes (KEGG) (19) pathway enrichment analyses, using the 'gene set function-functional enrichment-mRNA enrichment' module in the online Multifaceted Analysis Tool for Human Transcriptome (www.biocloudservice.com). Functional enrichment analyses were based on Fisher's exact test and $\mathrm{P}<0.05$ was selected as the threshold value.

PPI network and subnetwork module analyses. PPI pairs were predicted by the Search Tool for the Retrieval of Interacting Genes database (version 10.0; http://www.string-db.org/) (20) with a confidence score of $>0.4$. Using the obtained PPI pairs, the PPI network was constructed with Cytoscape version 3.2.0 (21). All nodes in the PPI network were DEGs. In the PPI network, the node score was calculated based on degree centrality. Nodes with a high score, which likely have an

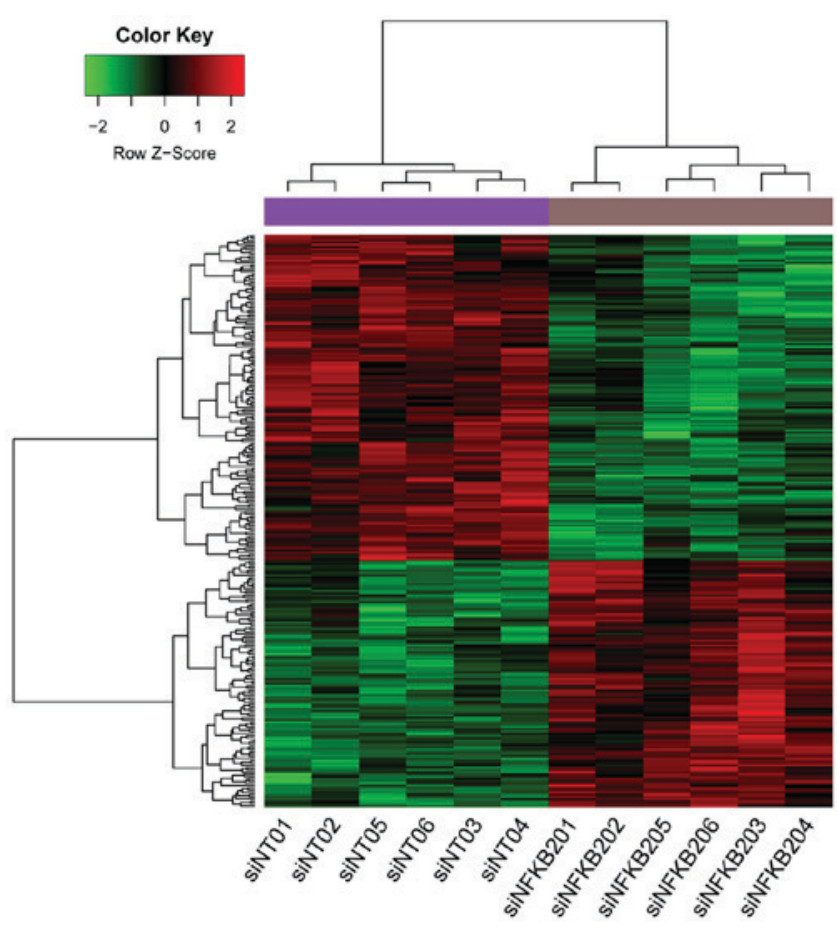

Figure 1. Heat map analysis of differentially expressed genes in the siNT and siNFKB2 groups. Red indicates high expression values and green indicates low expression values. si, small interfering RNA; siNT, non-targeting siRNA; NFKB2, nuclear factor- $\kappa$ B subunit 2; siNFKB2, NFKB2-targeting siRNA.

important role in the PPI network, were referred to as hub proteins. Proteins typically interact with other proteins in a module. Therefore, following PPI network analysis, significant modules from the PPI network were analyzed using the MultiContrast Delayed Enhancement (MCODE) version 1.4.2 (22) plugin in Cytoscape (23).

miRNA-target regulatory network analysis. miRNAs have a major role in the regulation of biological processes in organisms (24). Therefore, miRNAs that may target the identified DEGs were predicted using the 'gene-miRNA interaction information retrieval system' in miRWalk 2.0 (25) (http://mirwalk. uni-hd.de/), with a minimum seed length of seven and statistical significance set at $\mathrm{P}<0.05$. miRWalk is a comprehensive database that not only documents miRNA binding sites within the complete sequence of a gene, but also combines this information with a comparison of binding sites resulting from 12 existing miRNA-target prediction programs (25). In this study, the identified miRNA-target gene pairs required prediction by miRWalk, which included miRanda, miRDB 4.0, miRmap and TargetScan 6.2 databases. Predicted miRNAs were reviewed using the 'holistic view of validated disease-miRNA interactions' system in miRWalk 2.0 to select miRNAs that were confirmed to be associated with HL. These verified miRNA-target pairs were used to construct the regulatory network in Cytoscape (21).

\section{Results}

DEG screening. A total of 253 DEGs were identified following data preprocessing, which included 109 upregulated DEGs 
Table I. Kyoto Encyclopedia of Genes and Genomes pathway enrichment analysis for upregulated and downregulated DEGs.

A, Upregulated DEGs

\begin{tabular}{llll}
\hline ID & \multicolumn{1}{c}{ Name } & P-value & \multicolumn{1}{c}{ Genes } \\
\hline hsa04611 & Platelet activation & 0.03878 & PLA2G4F, FCGR2A, PRKG2, PRKG1 \\
hsa04380 & Osteoclast differentiation & 0.03953 & TYK2, GAB2, FCGR2A, MAPK10 \\
hsa04730 & Long-term depression & 0.04684 & PLA2G4F, PRKG2, PRKG1 \\
\hline
\end{tabular}

B, Downregulated DEGs

\begin{tabular}{|c|c|c|c|}
\hline ID & Name & P-value & Genes \\
\hline hsa04940 & Type I diabetes mellitus & 0.0003212 & CD80, HLA-DRB5, IL12B, HLA-DPB1, LTA \\
\hline hsa04672 & $\begin{array}{l}\text { Intestinal immune network } \\
\text { for } \operatorname{IgA} \text { production }\end{array}$ & 0.0004971 & $\begin{array}{l}\text { CD80, HLA-DRB5, TNFRSF17, AICDA, } \\
\text { HLA-DPB1 }\end{array}$ \\
\hline hsa05330 & Allograft rejection & 0.003062 & CD80, HLA-DRB5, IL12B, HLA-DPB1 \\
\hline hsa05166 & HTLV-I infection & 0.003913 & $\begin{array}{l}\text { WNT5A, CSF2, TLN1, HLA-DRB5, NFKB2, } \\
\text { BCL2L1, HLA-DPB1, LTA }\end{array}$ \\
\hline hsa04064 & $\mathrm{NF}-\kappa \mathrm{B}$ signaling pathway & 0.004905 & NFKB2, BCL2L1, BIRC2, PLAU, LTA \\
\hline hsa04060 & $\begin{array}{l}\text { Cytokine-cytokine } \\
\text { receptor interaction }\end{array}$ & 0.009454 & $\begin{array}{l}\text { CSF2, CCR } 4 \text {, TNFRSF17, TNFRSF8, } \\
\text { TNFRSF14, IL12B, LTA }\end{array}$ \\
\hline hsa05145 & Toxoplasmosis & 0.01411 & $\begin{array}{l}\text { HLA-DRB5, BCL2L1, IL12B, HLA-DPB1, } \\
\text { BIRC2 }\end{array}$ \\
\hline hsa05168 & Herpes simplex infection & 0.01472 & $\begin{array}{l}\text { NECTIN1, HLA-DRB5, TNFRSF14, IL12B, } \\
\text { HLA-DPB1, LTA }\end{array}$ \\
\hline hsa04062 & Chemokine signaling pathway & 0.0157 & $\begin{array}{l}\text { CCL22, CCR } 4, \text { PTK2B, HCK, GRK2, } \\
\text { PRKCD }\end{array}$ \\
\hline hsa04514 & Cell adhesion molecules (CAMs) & 0.02597 & $\begin{array}{l}\text { ALCAM, CD80, NECTIN1, HLA-DRB5, } \\
\text { HLA-DPB1 }\end{array}$ \\
\hline hsa05332 & Graft-versus-host disease & 0.02806 & CD80, HLA-DRB5, HLA-DPB1 \\
\hline hsa05323 & Rheumatoid arthritis & 0.03255 & CSF2, CD80, HLA-DRB5, HLA-DPB1 \\
\hline hsa05202 & Transcriptional misregulation in cancer & 0.04407 & CSF2, REL, RARA, BCL2L1, PLAU \\
\hline
\end{tabular}

DEGs, differentially expressed genes.

and 144 downregulated DEGs. The heat map clearly indicated the distinct separation of the knockdown and control groups (Fig. 1).

Functional enrichment analyses. The upregulated DEGs were significantly enriched in 'platelet activation', 'osteoclast differentiation' and 'long-term depression' KEGG pathways, while downregulated DEGs were predominantly enriched in 'intestinal immune network for IgA production', 'HTLV-I infection' and 'NF- $\mathrm{B}$ signaling pathway' KEGG pathways (Table I).

Additionally, upregulated DEGs were significantly enriched in $10 \mathrm{GO}$ terms, including 'integral component of membrane', 'plasma membrane' and 'Golgi apparatus' terms (Fig. 2A). Downregulated DEGs were significantly enriched in 53 GO terms, including 'protein binding', 'cytoplasm', 'transcription factor activity' and 'sequence-specific DNA binding'. The top 15 downregulated GO terms are presented in Fig. 2B.
PPI network analysis. The PPI network included 142 nodes and 220 PPI pairs (Fig. 3). Based on degree centrality analysis, 8 upregulated and 12 downregulated DEGs were determined to be hub genes (Table II). KEGG pathway enrichment analysis was subsequently performed to investigate the function of the 20 identified hub genes. As presented in Table III, these hub genes were significantly enriched in 14 KEGG pathways. For instance, B-cell lymphoma 2-like 1 (BCL2L1) was significantly enriched in 'NF- $\kappa \mathrm{B}$ signaling pathway' and 'Jak-STAT signaling pathway'. Colony-stimulating factor 2 (CSF2) was significantly enriched in 'transcriptional misregulation in cancer', 'TNF signaling pathway' and 'Jak-STAT signaling pathway'. Tyrosine kinase 2 was significantly enriched in 'Jak-STAT signaling pathway', 'toxoplasmosis', 'Epstein-Barr virus infection' and 'osteoclast differentiation'. CD44 antigen was enriched in 'Epstein-Barr virus infection'.

Subnetwork module analysis. Module analysis identified three modules (termed A, B and C; Fig. 4). Module A was 
$\mathbf{A}$

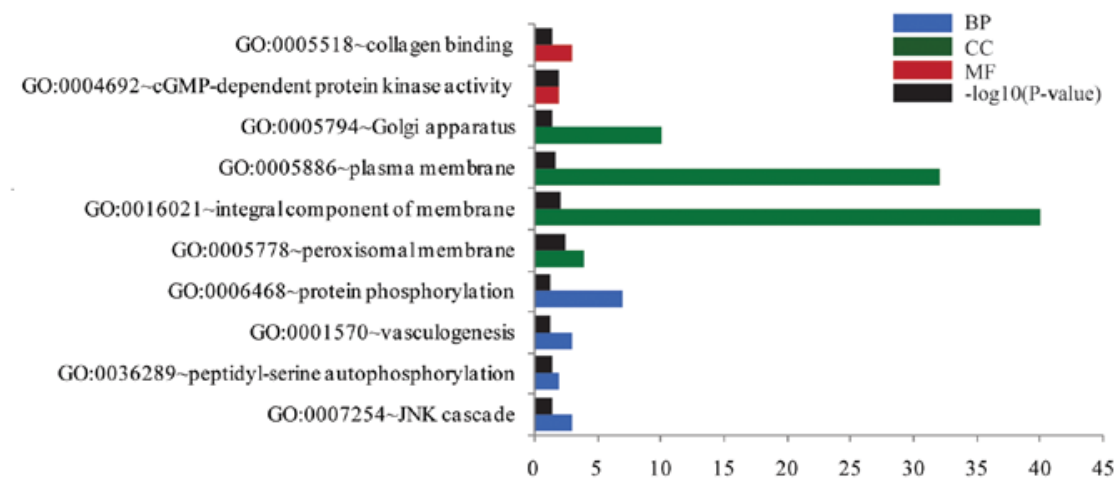

B

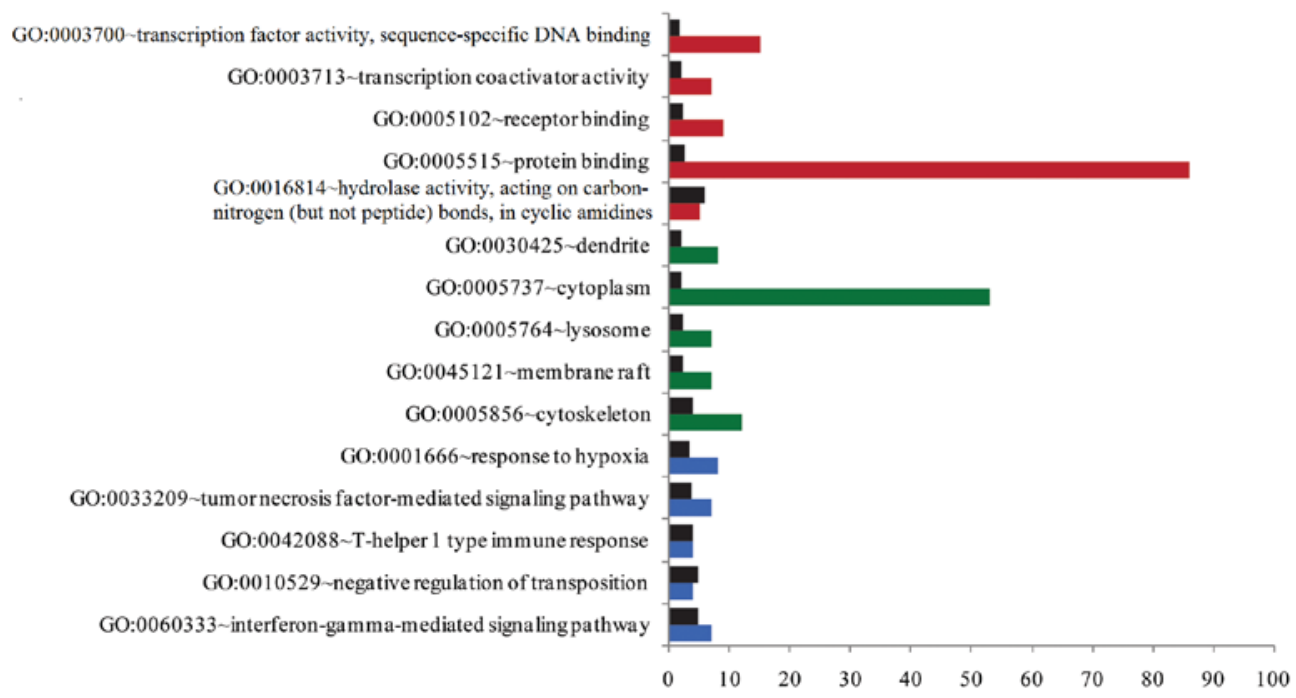

Figure 2. GO enrichment analysis for DEGs in BP, CC and MF terms. The number on the $\mathrm{x}$-axis indicates the enriched count of differentially expressed genes. (A) Enriched BP, CC and MF GO terms in the upregulated DEGs. (B) Top 15 enriched GO terms (BP, CC and MF) for downregulated DEGs. GO, Gene Ontology; DEGs, differentially expressed genes; BP, biological process; CC, cellular component; MF, molecular function.

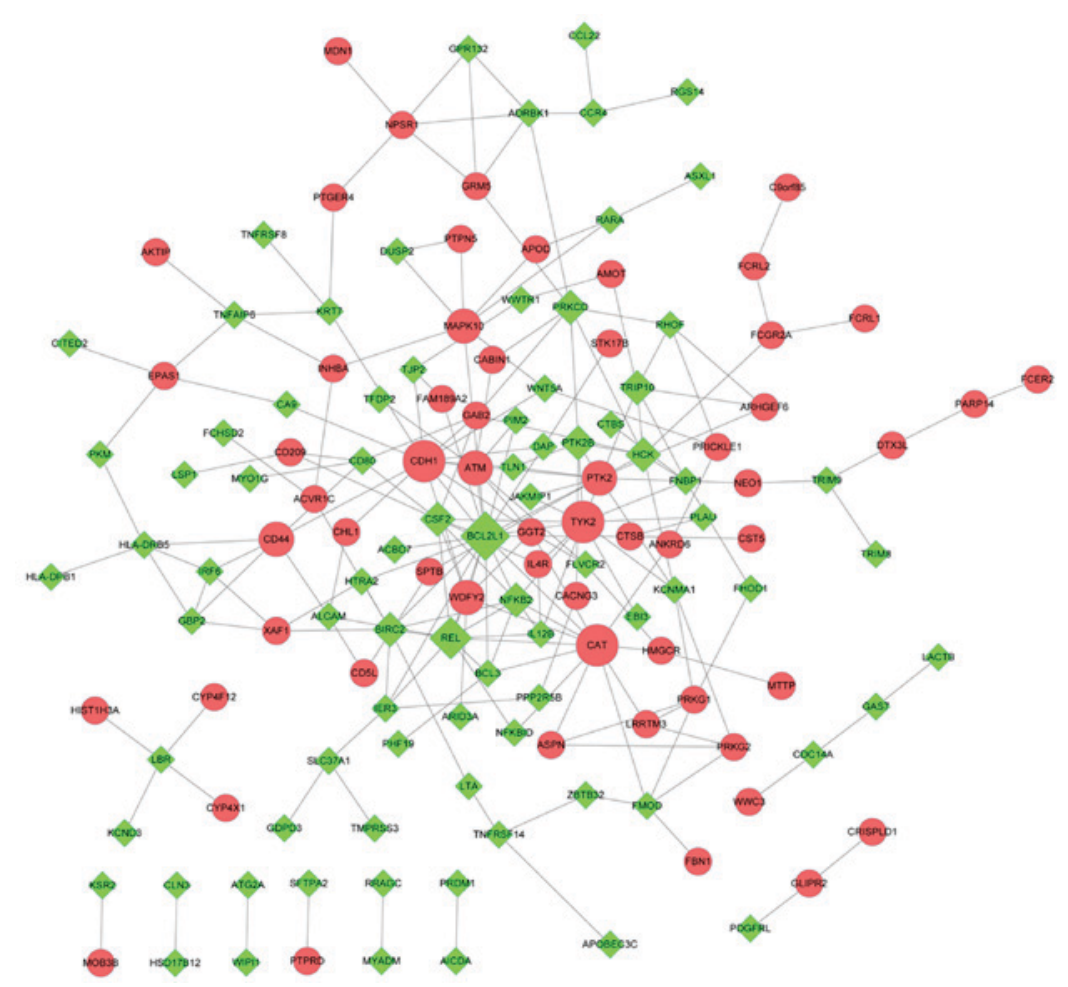

Figure 3. Protein-protein interaction network analysis of DEGs. Red circles represent upregulated DEGs and green diamonds represent downregulated DEGs. DEGs, differentially expressed genes. 
Table II. Degree centrality analysis for 20 hub genes.

\begin{tabular}{llc}
\hline Node & Description & Degree \\
\hline BCL2L1 & Downregulated & 19 \\
CDH1 & Upregulated & 13 \\
CAT & Upregulated & 12 \\
TYK2 & Upregulated & 12 \\
REL & Downregulated & 11 \\
PTK2 & Upregulated & 10 \\
CSF2 & Downregulated & 9 \\
HCK & Downregulated & 8 \\
BIRC2 & Downregulated & 8 \\
NFKB2 & Downregulated & 8 \\
WDFY2 & Upregulated & 7 \\
MAPK10 & Upregulated & 7 \\
PRKCD & Downregulated & 7 \\
ATM & Upregulated & 6 \\
TRIP10 & Downregulated & 6 \\
CD44 & Upregulated & 6 \\
PTK2B & Downregulated & 6 \\
FMOD & Downregulated & 5 \\
FNBP1 & Downregulated & 5 \\
IER3 & Downregulated & 5 \\
\hline
\end{tabular}

comprised of 5 nodes, 10 interaction pairs and was enriched in 'amyotrophic lateral sclerosis (ALS)' and ' $N F-\kappa B$ signaling pathway' KEGG pathways. There were 4 nodes and 6 interaction pairs in module B, without KEGG pathway enrichment. Module $\mathrm{C}$ contained 4 nodes and 6 interaction pairs and was enriched in the 'hematopoietic cell lineage' KEGG pathway.

miRNA-target regulatory network analysis. The miRNA-target regulatory network was constructed with 32 nodes and 29 PPI pairs (Fig. 5). Among the 32 nodes, there were 16 upregulated DEGs, 10 downregulated DEGs and 6 miRNAs, which included let-7a-5p, miR-9-5p, miR-155-5p, miR-135a-5p, miR-17-5p and miR-375.

\section{Discussion}

In the present study, a total of 109 upregulated and 144 downregulated DEGs were identified between the NFKB2 knockdown and control groups. Pathway enrichment analysis revealed that BCL2L1 was significantly enriched in the $\mathrm{NF}-\kappa \mathrm{B}$ signaling pathway, and CSF2 and BCL2L1 were enriched in the Jak-STAT signaling pathway. Additionally, BCL2L1 and CSF2 were hub genes in the PPI network. Prediction of miRNA-target interactions identified 29 miRNA-target pairs involving in 6 miRNAs (let-7a-5p, miR-9-5p, miR-155-5p, miR-135a-5p, miR-17-5p and miR-375). The findings of the present study may provide guidelines for understanding the regulatory mechanism of NFKB2 and contribute to the identification of therapeutic targets for HL.

BCL2L1 was demonstrated to be downregulated in HL cell lines following NFKB2 knockdown. This gene is a member of the Bcl-2 family and is a vital apoptosis-mediating gene that encodes both an antiapoptotic and proapoptotic splice variant (26). Additionally, in the present study, module analysis revealed that BCL2L1 interacts with NFKB2 in module $A$, which indicated that knockdown of NFKB2 may suppress BCL2L1 expression and subsequently regulate HL cell apoptosis. Notably, this result was consistent with the findings of de Oliveira et al (10), who reported that NFKB2 knockdown may downregulate BCL2L1 expression. Furthermore, BCL2L1 was enriched in the $\mathrm{NF}-\kappa \mathrm{B}$ signaling pathway in the present study. The $\mathrm{NF}-\kappa \mathrm{B}$ signaling pathway has been associated with the progression of several tumors, including $\operatorname{HL}(7,11)$. Therefore, it was speculated that BCL2L1 may be involved in $\mathrm{NF}-\kappa \mathrm{B}$-dependent control of HL cell survival.

In addition to BCL2L1, CSF2 was also downregulated in the NFKB2 knockdown group. CSF2 encodes a cytokine that has been reported to be involved in the pathogenesis of HL (27) and is highly expressed in HL cells (28). In accordance with these findings, the present study demonstrated that CSF2 was upregulated in HL control cells compared with HL cells with NFKB2 knockdown, indicating that NFKB2 may be involved in HL tumorigenesis through the upregulation of CSF2. KEGG pathway enrichment analysis revealed that CSF2 was enriched in the Jak-STAT signaling pathway. The Jak-STAT signaling pathway is regulated by a large array of cytokines and growth factors that induce proliferation or differentiation (29). Activation of the Jak-STAT signaling pathway is a hallmark of various B-cell lymphomas, including classical HL (30). Taken together, these data indicate that NFKB2 may be implicated HL development by regulating CSF2 expression, which in turn activates the Jak-STAT signaling pathway.

miRNAs regulate gene expression and cellular processes (24). They are also potential diagnostic and prognostic molecular markers in cancer, as well as targets for the development of highly specific therapies (31). Based on the DEGs identified in the present study, several miRNAs were predicted. Among these, miR-135a-5p has been previously reported to have critical involvement in HL cell survival by targeting Jak2 (32). Additionally, miR-155-5p has been recommended as a diagnostic marker in hematological tumors (33). Notably, dysregulated expression of miR-155 has been reported in various types of lymphoma (33). Differential expression of miR-9-5p has been demonstrated in hematological cell lines compared with normal lymphocyte populations (34). Furthermore, a target gene of miR-9-5p, PR/SET domain 1, was reported to be downregulated in HL cells (35), further indicating a potential role for miR-9-5p in HL. Therefore, NFKB2 may be implicated in HL development through interaction with these miRNAs.

In conclusion, the present study demonstrated that NFKB2 may be involved in the development of HL by interacting with several genes and miRNAs, including BCL2L1, CSF2, miR-135a-5p, miR-155-5p and miR-9-5p. Additionally, NFKB2-mediated modulation of the Jak-STAT and NF- $\kappa$ B signaling pathways may have a role in HL tumorigenesis. The genes, miRNAs and signaling pathways identified in the present study may serve as promising therapeutic targets for HL. Future experimental verification in vivo is required to confirm the findings of the present study. 
Table III. Kyoto Encyclopedia of Genes and Genomes pathway enrichment analysis for the 20 hub genes.

\begin{tabular}{llll}
\hline ID & \multicolumn{1}{c}{ Name } & \multicolumn{1}{c}{ P-value } & \\
\hline hsa05202 & Transcriptional misregulation in cancer & 0.0003731 & CSF2, PTK2, REL, BCL2L1, ATM \\
hsa04064 & NF-kB signaling pathway & 0.0007863 & NFKB2, BCL2L1, BIRC2, ATM \\
hsa05200 & Pathways in cancer & 0.001083 & PTK2, CDH1, NFKB2, BCL2L1, MAPK10, BIRC2 \\
hsa05145 & Toxoplasmosis & 0.001901 & TYK2, BCL2L1, MAPK10, BIRC2 \\
hsa04062 & Chemokine signaling pathway & 0.006877 & PTK2, PTK2B, HCK, PRKCD \\
hsa05169 & Epstein-Barr virus infection & 0.007294 & TYK2, CD44, NFKB2, MAPK10 \\
hsa04210 & Apoptosis & 0.007715 & BCL2L1, BIRC2, ATM \\
hsa05222 & Small cell lung cancer & 0.01415 & PTK2, BCL2L1, BIRC2 \\
hsa04912 & GnRH signaling pathway & 0.01611 & PTK2B, MAPK10, PRKCD \\
hsa05166 & HTLV-I infection & 0.01643 & CSF2, NFKB2, BCL2L1, ATM \\
hsa04668 & TNF signaling pathway & 0.02149 & CSF2, MAPK10, BIRC2 \\
hsa04380 & Osteoclast differentiation & 0.03187 & TYK2, NFKB2, MAPK10 \\
hsa04068 & FoxO signaling pathway & 0.03323 & CAT, MAPK10, ATM \\
hsa04630 & Jak-STAT signaling pathway & 0.03840 & TYK2, CSF2, BCL2L1
\end{tabular}
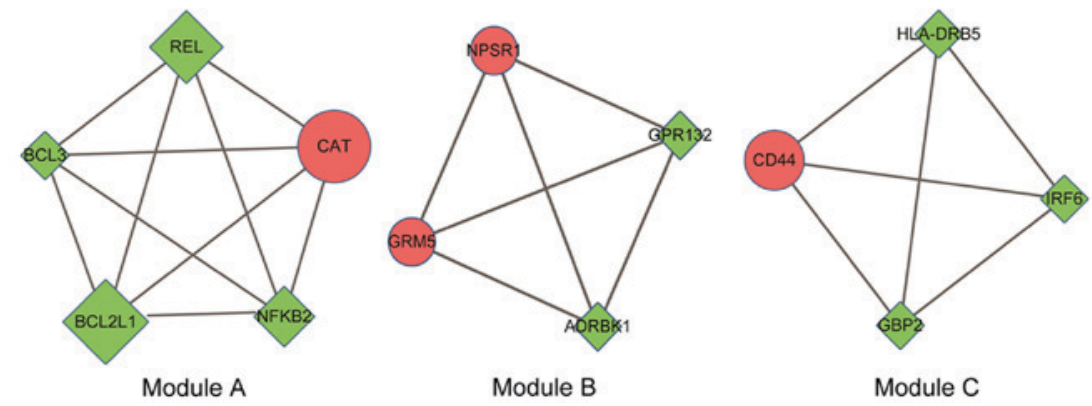

Figure 4. Subnetwork module analysis of DEGs identified three modules. Red circles represent upregulated DEGs and green diamonds represent DEGs. DEGs, differentially expressed genes.
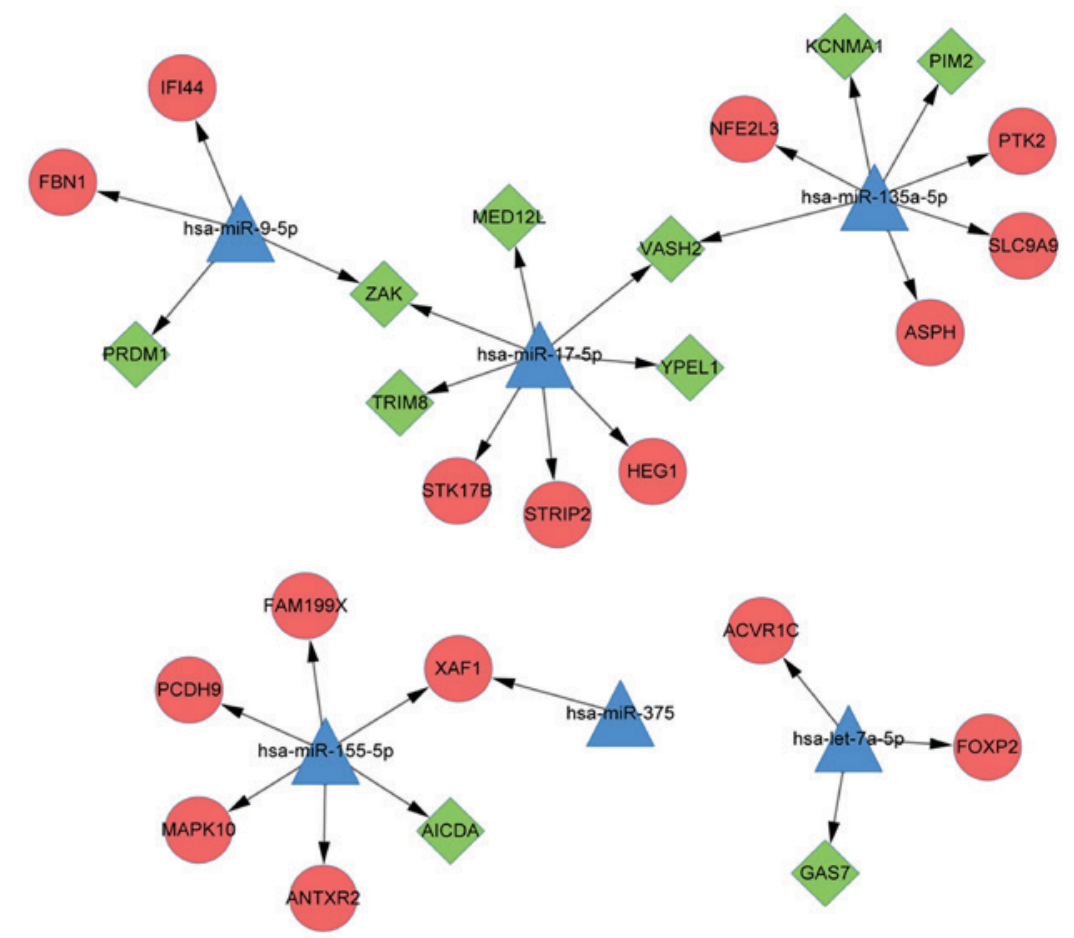

Figure 5. miRNA-target regulatory network analysis. Blue triangles represent miRNA, red circles represent upregulated DEGs, green diamonds represent downregulated DEGs and the arrows indicate the direction of regulation. miRNA, microRNA; DEGs, differentially expressed genes. 


\section{Acknowledgements}

Not applicable.

\section{Funding}

No funding was received.

\section{Availability of data and materials}

The datasets used and/or analyzed during the current study are available from the corresponding author on reasonable request.

\section{Authors' contributions}

YZ and LW conceptualized the study design. LT and SC acquired the data. LT, SC and WW performed the statistical analysis. YZ drafted the manuscript. LW revised the manuscript. All author read and approved the final manuscript.

\section{Ethics approval and consent to participate}

Not applicable.

\section{Consent for publication}

Not applicable.

\section{Competing interests}

The authors declare that they have no competing interests.

\section{References}

1. Wethal T, Lund MB, Edvardsen T, Fosså SD, Pripp AH, Holte H, Kjekshus J and Fosså A: Valvular dysfunction and left ventricular changes in Hodgkin's lymphoma survivors. A longitudinal study. Br J Cancer 101: 575-581, 2009.

2. Swerdlow SH, Campo E, Harris NL, Jaffe ES, Pileri SA, Stein H, Thiele J and Vardiman JW (eds): WHO classification of tumours of haematopoietic and lymphoid tissues. 4th edition. IARC Press, Lyon, 2008.

3. Connors JM: State-of-the-art therapeutics: Hodgkin's lymphoma. J Clin Oncol 23: 6400-6408, 2005.

4. Diehl V, Franklin J, Pfreundschuh M, Lathan B, Paulus U, Hasenclever D, Tesch H, Herrmann R, Dörken B, Müller-Hermelink HK, et al: Standard and increased-dose BEACOPP chemotherapy compared with COPP-ABVD for advanced Hodgkin's disease. N Engl J Med 348: 2386-2395, 2003.

5. Steidl C, Lee T, Shah SP, Farinha P, Han G, Nayar T, Delaney A, Jones SJ, Iqbal J, Weisenburger DD, et al: Tumor-associated macrophages and survival in classic Hodgkin's lymphoma. N Engl J Med 362: 875-885, 2010.

6. Heidenreich PA, Schnittger I, Strauss HW, Vagelos RH, Lee BK, Mariscal CS, Tate DJ, Horning SJ, Hoppe RT and Hancock SL: Screening for coronary artery disease after mediastinal irradiation for Hodgkin's disease. J Clin Oncol 25: 43-49, 2006.

7. Lim KH, Yang Y and Staudt LM: Pathogenetic importance and therapeutic implications of NF- $\kappa \mathrm{B}$ in lymphoid malignancies. Immunol Rev 246: 359-378, 2012.

8. Didonato JA, Mercurio F and Karin M: NF- $\mathrm{kB}$ and the link between inflammation and cancer. Immunol Rev 246: 379-400, 2012.

9. Jost PJ and Ruland J: Aberrant NF-kappaB signaling in lymphoma: Mechanisms, consequences, and therapeutic implications. Blood 109: 2700-2707, 2007.
10. de Oliveira KA, Kaergel E, Heinig M, Fontaine JF, Patone G, Muro EM, Mathas S, Hummel M, Andrade-Navarro MA, Hübner $\mathrm{N}$ and Scheidereit C: A roadmap of constitutive $\mathrm{NF}-\kappa \mathrm{B}$ activity in Hodgkin lymphoma: Dominant roles of p50 and 52 revealed by genome-wide analyses. Genome Med 8: 28, 2016.

11. Khan G: Epstein-Barr virus, cytokines, and inflammation: A cocktail for the pathogenesis of Hodgkin's lymphoma? Exp Hematol 34: 399-406, 2006.

12. Neri A, Fracchiolla NS, Migliazza A, Trecca D and Lombardi L: The involvement of the candidate proto-oncogene NFKB2/lyt-10 in lymphoid malignancies. Leuk Lymphoma 23: 43-48, 1996.

13. Neri A, Chang CC, Lombardi L, Salina M, Corradini P, Maiolo AT, Chaganti RS and Dalla-Favera R: B cell lymphoma-associated chromosomal translocation involves candidate oncogene lyt-10, homologous to NF-kappa B p50. Cell 67: 1075-1087, 1991

14. Irizarry RA, Hobbs B, Collin F, Beazer-Barclay YD, Antonellis KJ, Scherf U and Speed TP: Exploration, normalization, and summaries of high density oligonucleotide array probe level data. Biostatistics 4: 249-264, 2003.

15. Ihaka R and Gentleman R: R: A language for data analysis and graphics. J Comput Graphical Stat 5: 299-314, 1996.

16. Smyth GK: Limma: Linear models for microarray data. Bioinformatics Comput Biol Solut Using R Bioconductor: 397-420, 2005.

17. Wang L, Cao C, Ma Q, Zeng Q, Wang H, Cheng Z, Zhu G, Qi J, Ma H, Nian $\mathrm{H}$ and Wang Y: RNA-seq analyses of multiple meristems of soybean: Novel and alternative transcripts, evolutionary and functional implications. BMC Plant Biol 14: 169, 2014.

18. Ashburner M, Ball CA, Blake JA, Botstein D, Butler H, Cherry JM, Davis AP, Dolinski K, Dwight SS, Eppig JT, et al: Gene ontology: Tool for the unification of biology. The Gene Ontology Consortium. Nat Genet 25: 25-29, 2000.

19. Kanehisa M and Goto S: KEGG: Kyoto encyclopedia of genes and genomes. Nucleic Acids Res 28: 27-30, 2000.

20. von Mering C, Huynen M, Jaeggi D, Schmidt S, Bork P and Snel B: STRING: A database of predicted functional associations between proteins. Nucleic Acids Res 31: 258-261, 2003.

21. Shannon P, Markiel A, Ozier O, Baliga NS, Wang JT, Ramage D, Amin N, Schwikowski B and Ideker T: Cytoscape: A software environment for integrated models of biomolecular interaction networks. Genome Res 13: 2498-2504, 2003.

22. Bader GD and Hogue CW: An automated method for finding molecular complexes in large protein interaction networks. BMC Bioinformatics 4: 2, 2003.

23. Bandettini WP, Kellman P, Mancini C, Booker OJ, Vasu S, Leung SW, Wilson JR, Shanbhag SM, Chen MY and Arai AE: MultiContrast Delayed Enhancement (MCODE) improves detection of subendocardial myocardial infarction by late gadolinium enhancement cardiovascular magnetic resonance: A clinical validation study. J Cardiovasc Magn Reson 14: 83, 2012.

24. Hobert O: Gene regulation by transcription factors and microRNAs. Science 319: 1785-1786, 2008

25. Dweep H and Gretz N: miRWalk2.0: A comprehensive atlas of microRNA-target interactions. Nat Methods 12: 697, 2015.

26. Sillars-Hardebol AH, Carvalho B, Beliën JA, de Wit M, Delis-van Diemen PM, Tijssen M, van de Wiel MA, Pontén F, Fijneman RJ and Meijer GA: BCL2L1 has a functional role in colorectal cancer and its protein expression is associated with chromosome 20q gain. J Pathol 226: 442-450, 2012.

27. Steidl C, Connors JM and Gascoyne RD: Molecular pathogenesis of Hodgkin's lymphoma: Increasing evidence of the importance of the microenvironment. J Clin Oncol 29: 1812-1826, 2011.

28. Brase JC, Johannes M, Schlomm T, Fälth M, Haese A, Steuber T, Beissbarth T, Kuner R and Sültmann H: Circulating miRNAs are correlated with tumor progression in prostate cancer. Int J Cancer 128: 608-616, 2011.

29. Harrison DA, Mccoon PE, Binari R, Gilman M and Perrimon N: Drosophila unpaired encodes a secreted protein that activates the JAK signaling pathway. Genes Dev 12: 3252-3263, 1998.

30. Zahn M, Marienfeld R, Melzner I, Heinrich J, Renner B, Wegener S, Mießner A, Barth TF, Dorsch K, Brüderlein S and Möller P: A novel PTPN1 splice variant upregulates JAK/STAT activity in classical Hodgkin lymphoma cells. Blood 129: 1480-1490, 2017.

31. Iorio MV and Croce CM: MicroRNAs in cancer: Small molecules with a huge impact. J Clin Oncol 27: 5848-5856, 2009. 
32. Navarro A, Diaz T, Martinez A, Gaya A, Pons A, Gel B, Codony C, Ferrer G, Martinez C, Montserrat E and Monzo M: Regulation of JAK2 by miR-135a: Prognostic impact in classic Hodgkin lymphoma. Blood 114: 2945-2951, 2009.

33. Jurkovicova D, Magyerkova M, Kulcsar L, Krivjanska M, Krivjansky V, Gibadulinova A, Oveckova I and Chovanec M: miR-155 as a diagnostic and prognostic marker in hematological and solid malignancies. Neoplasma 61: 241-251, 2014.

34. Lawrie CH, Saunders NJ, Soneji S, Palazzo S, Dunlop HM, Cooper CD, Brown PJ, Troussard X, Mossafa H, Enver T, et al: MicroRNA expression in lymphocyte development and malignancy. Leukemia 22: 1440-1446, 2008.
35. Nie K, Gomez M, Landgraf P, Garcia JF, Liu Y, Tan LH, Chadburn A, Tuschl T, Knowles DM and Tam W: MicroRNA-mediated down-regulation of PRDM1/Blimp-1 in Hodgkin/Reed-Sternberg cells: A potential pathogenetic lesion in Hodgkin lymphomas. Am J Pathol 173: 242-252, 2008.

c) (i) (3) This work is licensed under a Creative Commons CY ${ }_{\mathrm{EO}} \mathrm{NO}$ Attribution-NonCommercial-NoDerivatives 4.0 International (CC BY-NC-ND 4.0) License. 